\title{
Estudo Comparativo de Fatores Prognósticos em Portadores de Carcinoma Não-Pequenas Células de Pulmão: Sobrevida Superior a Cinco Anos e Inferior a Um Ano \\ Comparative Study of Prognostic Factors Between two Groups of Patients With Non-Small Cell Lung Cancer: Survival Above Five Years and Below One Year
}

Sérgio Jamnik', Ilka Lopes Santoro', Eliana Lourenço Borges ${ }^{2}$, César Uehara ${ }^{3}$, Vilmer Vieira da Silva ${ }^{4}$

\section{Resumo}

Objetivo: verificar as diferenças dos fatores prognósticos de dois grupos distintos de portadores de carcinoma nãopequenas células de pulmão: sobrevida superior a cinco anos e sobrevida inferior ou igual a um ano. Métodos: vinte e oito pacientes com maior sobrevida e 182 pacientes com menor sobrevida avaliados dos prontuários do ambulatório de Onco-Pneumologia da Unifesp. Foram avaliados dados clínicos, hábito tabágico, peso, estádio, tratamento e laboratoriais (CEA e DHL). Resultados: os fatores que influenciaram a sobrevida foram a capacidade funcional, doença localizada, perda de peso, DHL, CEA e a cirurgia. Conclusão: a avaliação destes fatores permite a escolha do melhor tratamento para cada paciente.

Palavras-chave: Carcinoma pulmonar de células não-pequenas; Prognóstico; Sobrevida

${ }^{1}$ Médicos do ambulatório de Onco-Pneumologia da Unifesp, Doutores em Ciências pela Unifesp ${ }^{2}$ Pós-graduanda da Disciplina de Pneumologia

${ }^{3}$ Professor Adjunto da Disciplina de Pneumologia e Chefe do setor de Onco-Pneumologia da Unifesp

${ }^{4}$ Médico do ambulatório de Onco-Pneumologia da Unifesp

Endereço para Correspondência: Sérgio Jamnik. Rua Jaspe, 32 - apto. 121 - Aclimação - São Paulo (SP), Brasil - CEP: 01531-060.

E-mail: sergio@pneumo.epm.br 


\section{INTRODUÇÃO}

O carcinoma do pulmão é um dos cânceres de maior incidência, sendo a principal causa de morte relacionada ao câncer no mundo. Nos Estados Unidos, há registro de mais de 160.000 mortes/ano e, no Brasil, mais de 20.000 mortes/ano ${ }^{1,2,3}$. Devido à dificuldade para 0 diagnóstico precoce, a maioria apresenta estádios III e IV no momento do diagnóstico. Somente um terço destes pacientes submete-se à retirada total cirúrgica do tumor e, portanto, a maioria não é candidata a tratamento curativo. A maioria dos casos necessita de tratamentos paliativos: quimioterapia, radioterapia ou ambos $^{4}$. Diferentemente de outros cânceres, apenas um pequeno número de pacientes sobrevive mais de cinco anos $^{4-5}$. Esta porcentagem chega a $15 \%$ nos Estados Unidos. Uma série de fatores prognósticos (estadiamento, tipo histológico, marcadores tumorais, entre outros) parece influenciar no tempo de sobrevida, alguns de maneira mais concreta do que outros ${ }^{5,6}$.

Neste trabalho, foram comparados os fatores prognósticos entre uma população com sobrevida superior a cinco anos e uma com sobrevida inferior ou igual a um ano de um hospital universitário, uma vez que a literatura, apesar do grande número de artigos, continua controversa em relação aos fatores que levam a melhor e a pior prognóstico ${ }^{6}$. Isto permitiria um melhor conhecimento em direção a individualização do tratamento de cada paciente.

\section{MÉTODOS}

Foram avaliados os pacientes do ambulatório de Onco-Pneumologia da Unifesp, portadores de carcinoma broncogênico não-pequenas células, cuja sobrevida foi superior a cinco anos e inferior ou igual a um ano e que tiveram óbitos confirmados. Isto permitiria comparar os pacientes de melhor e pior prognóstico desse ambulatório. Foram analisados pacientes que ingressaram no ambulatório a partir do ano de 1986 até o ano 2000, portanto um período de acompanhamento de cinco anos. A obtenção dos dados foi através dos prontuários desse ambulatório no início do ano de 2006. No grupo de sobrevida inferior ou igual a um ano, ingressaram 182 pacientes e, no grupo de sobrevida superior a cinco anos, 28 pacientes. Considerou-se o início da contagem da sobrevida a data do diagnóstico do anatomopatológico. Todos os pacientes tinham diagnóstico histopatológico da doença. Avaliou-se a idade, sexo, sinais e sintomas, tipo histológico, capacidade funcional (índice de Karnofsky), hábito de fumar, índice maços/ano, estadiamento e tipo de tratamento. Também os seguintes exames laboratoriais: antígeno carcinoembrionário (CEA - nanogramas/ml) e desidrogenase lática (DHL-U/L).

Para facilitar a avaliação, os estadiamentos Ia, Ib, IIa, IIb e IIIa foram agrupados com a denominação doença precoce e os estadiamentos IIIb e IV doença avançada.

Em relação ao tratamento, os pacientes foram agrupados em três tipos: cirurgia, paliativo e suporte. Do grupo da cirurgia, participaram aqueles doentes que se submeteram à ressecção do tumor isoladamente ou associada à quimioterapia e/ou radioterapia e suporte. Do paliativo, aqueles doentes que só se submeteram à quimioterapia e/ou radioterapia e suporte. Do suporte, aqueles pacientes que usaram apenas tratamentos sintomáticos como dor e dispneia.

Este trabalho foi submetido e aprovado pela Comissão de Ética desta Instituição.

Os resultados dos dois grupos foram comparados, sendo utilizado o teste do Qui-quadrado $\chi^{2}$ ou teste exato de Fisher para variáveis nominais, e teste $t$ de Student para variáveis contínuas. Foram calculados o risco relativo (RR) e o intervalo de confiança 95\% (IC 95\%). Em todos os casos, o nível de significância para a rejeição da hipótese de nulidade foi igual ou menor do que 0,05 .

\section{RESULTADOS}

Entre o período de 1986 até 2000, participaram do ambulatório 1.201 pacientes. Destes, 31(2,6\%) sobreviveram mais de cinco anos, sendo 28 do tipo histológico carcinoma não-pequenas células. Dos 219 $(18,2 \%)$ que sobreviveram menos de um ano com óbito comprovado neste período, 182 eram carcinoma nãopequenas células. No primeiro grupo, $19(67,9 \%)$ eram homens e, no segundo grupo, 144 (79,1\%). Não houve diferença significante entre os grupos. Também não houve diferença entre os grupos quando comparados: idade, hábito de fumar e maços/ano. Já a diferença foi estatisticamente significante quando comparada a capacidade funcional $(\mathrm{p}<0,0001)$ (tabela 1$)$.

Entre os sinais e sintomas, a diferença entre os grupos foi somente significante na dor torácica $(\mathrm{p}=0,04)$, apesar de que, em muitos deles, houve uma tendência a uma maior frequência nos pacientes com menor sobrevida (tabela 1).

A média da perda de peso relatada pelos pacientes inicialmente não mostrou diferença significante (sobrevida maior do que cinco anos $=8,20 \pm 4,71 \mathrm{~kg} \mathrm{e}$ sobrevida inferior ou igual a um ano $=9,78 \pm 6,15 \mathrm{~kg}$ ). Em relação à porcentagem de pacientes que perderam peso, no seguimento, $35,7 \%$ ocorreram no grupo com 
sobrevida superior a cinco anos e $64,3 \%$ no grupo inferior ou igual a um ano, essa diferença foi muito significante $(\mathrm{p}=0,0029)$.

$\mathrm{O}$ número de pacientes que apresentou anorexia foi superior entre os pacientes com sobrevida inferior $(40,1 \%)$ em relação aos pacientes com sobrevida superior (25\%), apesar da diferença não ser significante.

A maior parte dos pacientes com sobrevida superior a cinco anos foi agrupada como doença precoce $(89,3 \%)$, ao contrário dos pacientes com sobrevida inferior ou igual a um ano que foram agrupados com doença avançada (94,5\%). Essa diferença foi extremamente significante $(\mathrm{p}=0,0001)$ (tabela 2$)$.

A distribuição por tipo histológico entre os sexos e entre os dois grupos foi semelhante, com exceção das mulheres do grupo sobrevida superior a cinco anos, em que houve um predomínio do tipo histológico de adenocarcinoma, mas que não foi estatisticamente significante $(\mathrm{p}=0,071)$ (tabela 3$)$.

Os valores médios do CEA foram inferiores no grupo de maior sobrevida $(2,72 \pm 2,33)$ em relação ao grupo de menor sobrevida $(26,87 \pm 56,26)$. Ocorreu o mesmo em relação aos valores médios do DHL, respectivamente, $208,72 \pm 113,39$ e 313,98 $\pm 185,39$. As diferenças foram significantes (tabela 4). Chama a atenção que os valores do CEA e DHL em sua grande maioria $(100 \%$ e $95,7 \%)$ se apresentavam na faixa considerada normal, no grupo com sobrevida superior. Já esta porcentagem era inferior no grupo com sobrevida menor $(63 \%$ e $76,5 \%)$. Essa diferença foi

Tabela 1. Características gerais do paciente e sinais e sintomas principais segundo a sobrevida

\begin{tabular}{|c|c|c|c|c|}
\hline & $\begin{array}{c}\text { Superior a } 5 \\
\text { anos }\end{array}$ & $\begin{array}{c}\text { Inferior ou igual } \\
\text { a } 1 \text { ano }\end{array}$ & $\mathbf{p}$ & RR (IC 95\%) \\
\hline Masculino & $19(67,8 \%)$ & $144(79,1 \%)$ & & \\
\hline Idade média \pm DP & $59,60 \pm 9,43$ & $62,34 \pm 12,25$ & & \\
\hline Índice Karnofsky (mediana|) & 100 & 70 & $<0,001$ & \\
\hline Índice Karnofsky (média) & $95 \pm 7,45$ & $71,04 \pm 17,09$ & & \\
\hline Índice Karnofsky (IC 95\%) & 92,11 a 97,89 & 64,41 a 73,66 & & \\
\hline Maços/ano (média) \pm DP & $44,10 \pm 19,97$ & $54,02 \pm 32,65$ & & \\
\hline Tosse & $29(67,8 \%)$ & $123(67,6 \%)$ & & \\
\hline Dispneia & $10(35,7 \%)$ & $99(54,3 \%)$ & & \\
\hline Dor torácica & $8(28,6 \%)$ & $101(55,4 \%)$ & 0,04 & $0,34(0,17$ a 0,73$)$ \\
\hline Anorexia & $7(25 \%)$ & $73(40,1 \%)$ & & \\
\hline Pacientes com perda de peso & $10(35,7 \%)$ & $121(66,5 \%)$ & 0,0029 & $2,98(1,41$ a 6,14$)$ \\
\hline
\end{tabular}

$\mathrm{DP}=$ desvio padrão

$\mathrm{RR}=$ risco relativo

Tabela 2. Estadiamento entre os grupos segundo a sobrevida

\begin{tabular}{l|c|c}
\hline & Superior a $\mathbf{5}$ anos & Inferior ou igual a $\mathbf{1}$ ano \\
\hline Doença precoce & $25(89,3 \%)$ & $10(5,5 \%)$ \\
\hline Doença avançada & $3(10,7 \%)$ & $172(94,5 \%)$ \\
\hline
\end{tabular}

$\mathrm{p}<0,0001 ; \mathrm{RR}=41,67 ; \mathrm{IC} 95 \%=13,31$ a 130,40

Tabela 3. Tipo histológico e sexo segundo os grupos

\begin{tabular}{|c|c|c|c|c|}
\hline & \multicolumn{2}{|c|}{ Superior a 5 anos } & \multicolumn{2}{|c|}{ Inferior ou igual a 1 ano } \\
\hline & Homens & Mulheres & Homens & Mulheres \\
\hline Adenocarcinoma & $7(36,8 \%)$ & $7(77,8 \%)$ & $58(40,3 \%)$ & $18(47,4 \%)$ \\
\hline Carcinoma espinocelular & $10(52,6 \%)$ & $1(11,1 \%)$ & $72(50,0 \%)$ & $17(44,7 \%)$ \\
\hline Carcinoma indiferenciado & 0 & 0 & $10(6,9 \%)$ & $2(5,3 \%)$ \\
\hline Carcinoma grande células & $2(10,5 \%)$ & 0 & $4(2,8 \%)$ & $1(2,6 \%)$ \\
\hline Outros & $2(10,5 \%)$ & $1(11,1 \%)$ & 0 & 0 \\
\hline Total & 19 & 9 & 144 & 38 \\
\hline
\end{tabular}


estatisticamente significante tanto para o CEA $(\mathrm{p}<0,0001)$ e DHL $(\mathrm{p}=0,0003)$.

Entre os pacientes com sobrevida superior a cinco anos, $67,9 \%$ (19), e sobrevida inferior ou igual a um ano, $8,24 \%$ foram submetidos à cirurgia com o objetivo de cura. Já no primeiro grupo, $10,80 \%$ e, no segundo grupo, $24,73 \%$ só receberam suporte de vida. Essa diferença foi estatisticamente significante quando comparados os dois grupos em relação à realização da cirurgia $(\mathrm{p}<0,0001)($ tabela 5).

\section{DISCUSSÃO}

Raros pacientes de carcinoma pulmonar sobrevivem acima de cinco anos, uma vez que o diagnóstico é tardio, sendo que a grande maioria se apresenta como estadiamentos iniciais IIIb e IV. Neste serviço, até o momento do fim da coleta dos dados, este grupo representava 2,6\% dos pacientes. Neste período nos Estados Unidos, essa porcentagem chegava a 15\%5. Em outros países como: Inglaterra, Escócia, Dinamarca e a Europa toda, essa porcentagem atinge, respectivamente, $6 \%, 6 \%, 6,1 \%$ e $9,5 \% 5$. Existem vários fatores prognósticos que influenciam a sobrevida desses pacientes, incluindo os clínicos, estádio do tumor, índices patológicos e bioquímicos ${ }^{7}$.

As informações foram obtidas de um ambulatório universitário do ano de 1986 até o final de 2000. Isso permitiu que todos os pacientes, inclusive os últimos, ao participarem do estudo, tivessem um acompanhamento de pelo menos cinco anos. A maioria dos pacientes desse ambulatório é moradora da cidade de São Paulo, do interior do estado e de alguns outros estados.
O número de pacientes estudados ao todo é grande, mas como era de se esperar ele se torna em número bem menor $(\mathrm{N}=28)$ ao estudar aqueles com sobrevida superior a cinco anos e portadores de carcinoma nãopequenas células, o que pode dificultar a análise dos dados. Outra crítica a ele é que os dados estudados foram retrospectivos e abrangiam um período longo da década de 1980 até o ano de 2000. Esta última crítica se suaviza, uma vez que ocorreram nos dois grupos homogeneamente.

Entre os fatores clínicos, não se observou diferença entre o sexo e a idade em trabalho descrito no nosso meio ${ }^{7}$. Já na literatura internacional existe uma controvérsia, alguns considerando o sexo feminino como fator de melhor sobrevida. Estes a justificam pela maior expressão de receptores esteroides ER beta no câncer de pulmão de pacientes do sexo feminino em relação aos do sexo masculino ${ }^{8,9}$.

Os estudos mostram a capacidade funcional como um dos mais importantes fatores de previsão da sobrevida, mesmo após a correção do estadiamento e tratamento ${ }^{10,11}$. Neste trabalho, os valores da capacidade funcional foram superiores nos pacientes com sobrevida maior do que cinco anos e extremamente significantes, mostrando que valores maiores de capacidade funcional poderiam significar maiores chances de sobrevida mais longa.

Outro fator considerado importante, em relação à sobrevida, que a literatura mostra é a perda de peso inicial em relação ao peso habitual. Neste trabalho, $66,6 \%$ dos pacientes com sobrevida menor ou igual a um ano tiveram esta característica clínica maior do que o grupo com sobrevida superior a cinco anos e essa diferença foi muito significante $(\mathrm{p}=0,0035)^{7,11}$.

Tabela 4. Exames bioquímicos segundo os grupos

\begin{tabular}{l|c|c|c}
\hline & Superior a 5 anos & Inferior ou igual a 1 ano & $\mathrm{p}<0,0001$ \\
\hline CEA (média) & $2,72 \pm 2,33$ & $26,87 \pm 56,26$ & \\
\hline CEA (IC 95\%) & 1,80 a 3,64 & 14,86 a 38,89 & $\mathrm{p}=0,01$ \\
\hline DHL (média) & $208,72 \pm 113,39$ & $313,98 \pm 185,39$ & \\
\hline DHL (IC 95\%) & 186,00 a 258,44 & 275,53 a 352,45 & \\
\hline
\end{tabular}

CEA = antígeno carcinoembrionário

$\mathrm{DHL}=$ desidrogenase lática

Tabela 5. Tratamento realizado entre os grupos

\begin{tabular}{l|c|c} 
& Superior a 5 anos & Inferior ou igual a 1 ano \\
\hline Cirurgia & $19(67,9 \%)$ & $15(8,2 \%)$ \\
\hline Paliativo & $6(21,4 \%)$ & $122(67,0 \%)$ \\
\hline Suporte & $3(10,7 \%)$ & $45(24,8 \%)$ \\
\hline
\end{tabular}

$\mathrm{p}<0,0001 ; \mathrm{RR}=8,94 ; \mathrm{IC} 95 \%=2,87$ a 27,84 
Apesar da literatura valorizar a anorexia como fator precursor da perda de peso e um fator preditor independente da diminuição da sobrevida, neste trabalho não houve diferença entre os dois grupos, embora haja uma tendência de maior número porcentual no grupo de sobrevida inferior ou igual a um ano ${ }^{7,10}$. Talvez um maior número de pacientes no grupo de maior sobrevida tornaria essa diferença significante.

O estádio da doença no diagnóstico representa um dos mais poderosos determinantes do prognóstico no carcinoma de pulmão não-pequenas células, com os mais precoces estádios, tendo as melhores chances de maior sobrevida $^{12,13}$. Quanto maior o valor do índice T, N e M no estadiamento, pior a sobrevida ${ }^{14}$. Neste trabalho, os pacientes do grupo com sobrevida maior do que cinco anos, $87,1 \%$, apresentavam doença precoce e no grupo com sobrevida inferior ou igual a um ano, 93,8\% apresentavam doença avançada. Diferença essa significante $(\mathrm{p}=0,0001)$.

Em relação aos tipos histológicos, não existe certeza em relação à sobrevida. Os primeiros trabalhos mostravam que o carcinoma espinocelular apresentava maior sobrevida e menor taxa de recorrência do que os outros tipos histológicos, principalmente, nos estados precoces. Entretanto, estes achados não se confirmaram em trabalhos posteriores ${ }^{12,13-15,16}$. Neste trabalho, a distribuição dos tipos histológicos entre os dois grupos não apresentou diferenças ${ }^{17}$.

A literatura mostra que pacientes com níveis elevados de CEA e DHL pré-tratamento apresentam em geral uma pior sobrevida ${ }^{18,19,20,21}$. Os níveis elevados são mais frequentemente encontrados em idade mais elevada, sexo masculino, maior tamanho tumoral, ressecção incompleta e estádio avançado ${ }^{22,23}$. Neste trabalho, os maiores valores foram encontrados no grupo com sobrevida inferior ou igual a um ano.

A maior parte dos pacientes com sobrevida superior a cinco anos $(67,9 \%)$ foi submetida à cirurgia com intuito curativo e apenas $6,8 \%$ do grupo com sobrevida inferior ou igual a um ano foram submetidos à tal cirurgia. Isso ocorre porque o grupo com sobrevida superior a cinco anos apresenta estádios mais precoces, com doenças mais localizadas e capacidade funcional melhor. Isso permite que os pacientes consigam as condiçôes adequadas para a cirurgia, que o tumor seja tecnicamente ressecável e que sua sobrevida seja maior do que cinco anos após a diagnóstico ${ }^{24}$.

Mais recentemente, com o progresso da biologia molecular, aumentou o entendimento dos mecanismos relatados com a iniciação e progressão dos cânceres. Como consequência, estão desenvolvendo anticorpos monoclonais e pequenas moléculas, que interferem com alvos moleculares específicos, como: receptores dos fatores de crescimento, proteínas do ciclo celular, que são moduladores da apoptose, angiogênese e invasão celular. Com os novos métodos diagnósticos, tem-se observado alteraçôes genéticas específicas que podem elucidar a quimiossensibilidade e resistência, e a melhor seleção de drogas. Todo este desenvolvimento que em poucos anos melhorará a sobrevida. Neste trabalho, infelizmente não foi possível realizar esta avaliação, uma vez que a maioria deste desenvolvimento não existia no estudo desses doentes ${ }^{25}$.

Concluiu-se que os principais fatores de melhor prognóstico que ocorreram nos pacientes do grupo com sobrevida superior a cinco anos foram: maiores valores da capacidade funcional inicial, menor perda de peso inicial, doenças mais localizadas, menores valores iniciais do CEA e DHL e cirurgia com objetivo curativo.

\section{Declaração de conflito de interesses: nada a declarar.}

\section{REFERÊNCIAS}

1. Ahmedin JA, Siegal R, Ward E, Murray T, Xu J, Thun MJ, et al. Cancer statistics, 2007. CA Cancer J Clin. 2007;57(1):43-66.

2. Instituto Nacional do Câncer. Estimativas 2005: incidência de câncer no Brasil [monografia na Internet]. Rio de Janeiro: Instituto Nacional do Câncer; 2005 [citado em 2005 Out 20]. Disponível em: www.inca.gov.br/estimativa/2005.

3. Boing AF, RossiTF. Tendência temporal e distribuição espacial da mortalidade por câncer de pulmão no Brasil entre $1979 \mathrm{e}$ 2004: magnitude, padróes regionais e diferenças entre sexos. J Bras Pneumol. 2007;33(5):544-51.

4. Jamnik S, Tadokoro H, Segreto RA, Costa QJ, Segreto HRC, Yamauti SM, et al. Carcinoma broncogênico: tratamento oncológico clínico e radioterapia. In: Nery LE, Fernandes ALG, Perfeito JA, editores. Guias de medicina ambulatorial e hospitalar Unifesp / Escola Paulista de Medicina: pneumologia. São Paulo: Manole; 2006. p. 511-28.

5. Butler CA, Darragh KM, Curro GP, Anderson WJA. Variation in lung cancer survival rates between countries: do differences in data reporting contribute? Resp Med. 2006;100(9):1642-6.

6. Forte V. Fatores de risco nas lobectomias realizadas para tratar o carcinoma brônquico. 2006;32(6):30-32.

7. Jamnik S, Santoro IL, Uehara C. Estudo comparativo dos fatores prognósticos entre os pacientes com maior e menor sobrevida em portadores de carcinoma broncogênico. J Pneumol. 2002;28(5):245-9.

8. Mollerup S, Jorgensen K, Berge G, Haugen A. Expression of estrogen receptors alpha and beta in human lung tissue and cell lines. Lung Cancer. 2002;37(2):153-9. 
9. Park N, Kubik A, Zatracikaf P, Krepelo E. Lung cancer in women. Lung cancer. 2005;48:1-9.

10. Hoang T, Xu R, Schiller JH. Clinical model to predict surviral in chemanaive patients with advanced non-small cell lung cancer treated with third generation chemotherapy regimens based on eastern cooperative oncology group data. J Clin Oncol. 2005;23(1):175-9.

11. Stanby KE. Prognostic factors for survival in patients with inoperable lung cancer. J Natl Cancer Inst. 1980;65(1):25-9.

12. AdebonojoSA, Bowser AN, Moritz DM, Corcaran PC. Impact of revised stage classification of lung cancer on survival. A military experience. Chest. 1999;115(6):1507-38.

13. Van Rens MT, de la Riviere AB, Elbers HR, van Den Bosch JM. Prognostic assessment of 2.361 patients who underwent pulmonary resection for non-small cell lung cancer, stage I, II and IIIA. Chest. 2000;117(2):374-9.

14. Sakao Y, Miyamoto H, Yamazaki A. Prognostic significance of metastasis to the highest mediastinal lymph node in non-small cell lung cancer. Ann Thorac Cardiovasc Surg. 2006;81(3):292-8.

15. Gail MH, Eagan RT, Feld R, Ginsberg R, Gogdell B. Prognostic factors in patients with resected stage I NSCLC: a report from the lung cancer study group. Cancer. 1984;54(9):1802-10.

16. Postoperative T1 No non-small cell lung cancer. Squamous versus nonsquamous recurrences the lung cancer study group. J Thorac Cardiovasc Surg. 1987;94(3):349-58.

17. Martini N, Bains MS, Burt ME. Incidence of local recurrence and second primary tumors in resected stage I lung cancer. J Thorac Cardiovasc Surg. 1995;109(2):120-8.

18. Okada M, Nishio W, Sakamoto T, Uchino K, Yuki T,
Nakagawa A, et al. Prognostic significance of perioperative serum carcinoembryonic antigen in non-small cell lung cancer: analysis of 1.000 consecutive resections for clinical stage I disease. Ann Thorac Surg. 2004;78(1):216-21.

19. Tomita M, Matsuzaki Y, Shimizu T, Hara M, Cuyabe T, Onitsuka T. Relationship between serum carcinoembryonic antigen level and $\mathrm{T}$ status in non-small-cell lung cancer. Anticancer Res. 2006;26(5B):3845-8.

20. Vansteenkeste JF, DeLeyn PR, Deneffe GJ, Stalpaert G, Nackaerts KL, Lerut TE, et al. Survival and prognostic factors in resected N2 non-small-cell lung cancer: a study of 140 cases. Ann Thorac Surg. 1997;63(5):1441-50.

21. Albain KS, Crowley JJ, Leblanc M, Liveingston RB. Survival determinants in extensive-stage non-small-cell lung cancer: the Southwest Oncology Group experience. J Clin Oncology. 1991;9(8):1618-26.

22. Matsuoka K, Sumitoma S, Nakashuma N, Nakajima D, Miraki N. Prognostic value of carcinoembryonic antigen and CYFRA 21-1 in patients with pathological stage I nonsmall-cell lung cancer. Eur J Cardiothorac Surg. 2007;32(3):435-9.

23. Hsu WH, Huang CS, Hsu HS, Huang WJ, Lee HC, Huang BS, et al. Preoperative serum carcinoembryonic antigen level is a prognostic factor in women with early non-smallcell lung cancer. Ann Thorac Surg. 2007;83(2):419-24.

24. Reilly BJ. Preparing for pulmonary resection: preoperative evaluation of patients. Chest. 1997;112 Suppl 4:S206-8.

25. Olaussen KA, Mountizios G, Soria JC. ERCC1 as a risk stratier in platinum-based chemotherapy for nonsmall-cell lung cancer. Curr Opin Pulm Med. 2007; 13(4):284-89.

\section{Abstract}

Objectives: verify the differences of prognostic factors between two distinct groups of patients with non-small cell lung cancer, whose survival was above five years or below one year. Methods: data evaluation of 28 patients with survival above five years and 182 patients with survival below one year from the medical archive of Unifesp Oncopneumology ambulatory of clinical data, tabagic habits, weight, stage, treatment and laboratorial data (CEA and LHD) were evaluated. Outcomes: Factors that influenced survival were performance status, localized disease, weight loss, LDH, CEA and surgery. Conclusion: the evaluation of these factors allows the choice of the best treatment for each patient.

Key words: Carcinoma, non-small-cell lung; Prognosis; Survivorship (Public Health) 